\title{
Urolume urethral wallstent in the treatment of detrusor sphincter dyssynergia
}

\author{
S Juma, S D Niku, P P Brodak, A C Joseph \\ Division of Urology, University of California, San Diego Medical Center, San Diego, \\ California and Spinal Cord Injury Unit, Veterans Affairs Medical Center, La Jolla, \\ California, USA.
}

\begin{abstract}
Neuropathic voiding dysfunction with detrusor sphincter dyssynergia (DSD) is a common complication of spinal cord injury (SCI) and multiple sclerosis. Sphincterotomy has been recommended for treatment of DSD if the patient lacks the ability to do intermittent self catheterization. We report our preliminary results of the Urolume urethral wallstent for the treatment of DSD in 10 patients. The insertion technique proved simple, with a short hospital stay and low morbidity; however, its long term safety has not yet been established.
\end{abstract}

Keywords: prosthesis; urethral wallstent; urethral obstruction; neuropathic bladder.

\section{Introduction}

Detrusor sphincter dyssynergia (DSD) is characterized by involuntary external sphincter contraction during an involuntary detrusor contraction causing high bladder outlet resistance. DSD is most often seen in patients with neurological lesions between the brainstem and sacral spinal cord. Fifty per cent of patients with DSD develop serious urological complications and renal function deterioration if proper treatment is not instituted. ${ }^{1}$ The therapeutic options include either facilitating urine storage or bladder emptying. Sphincterotomy ${ }^{2}$ has been the accepted treatment for DSD if the patient is unable to perform intermittent self catheterization.

Expandable stainless steel mesh stents were developed for use in the vascular system and recently have been used for treatment in benign prostatic hypertrophy and urethral strictures. ${ }^{3-6}$ We present our experience with the use of the Urolume urethral wallstent in a group of SCI patients.

\section{Patients and methods}

Patients with a neuropathic bladder and DSD were prospectively evaluated as part of an investigational protocol to evaluate the use of the Urolume urethral wallstent (American Medical Systems, Minnesota) in the treatment of DSD. Inclusion criteria included males older than 21 years of age with DSD and adequate detrusor contraction who were candidates for sphincterotomy. Males with bladder, prostate or urethral pathology were excluded. The protocol was approved by the university investigational review board and informed consents were obtained. Ten patients met the criteria and underwent placement of the Urolume urethral stent. The type and level of injury of the patients is shown in Table I. Four patients had undergone previous sphincterotomy and the remainder of the patients were managed by condom catheter alone. All patients had recurrent UTI and eight patients had autonomic dysreflexia. Preoperative evaluation included measurement of serum chemistries, blood urea nitrogen (BUN), creatinine, intravenous urogram, renal ultrasound, nuclear renal scan, cystoscopy and videourodynamics. All patients received prophylactic antibiotics pre-and postoperatively.

The Urolume urethral wallstent is a biomedical prosthesis made of a woven superalloy tubular mesh. The prosthesis is available in two lengths $(2 \mathrm{~cm}, 3 \mathrm{~cm})$ and can expand to $14 \mathrm{~mm}$ (42 French) diameter 
Table I Preoperative and postoperative urodynamic data of the patients. The postoperative data is at 12 months

\begin{tabular}{|c|c|c|c|c|c|c|c|}
\hline \multirow[t]{3}{*}{ Age } & \multirow[t]{3}{*}{ Level } & \multicolumn{3}{|c|}{ Preoperative urodynamics } & \multicolumn{3}{|c|}{ Postoperative urodynamics } \\
\hline & & & Max & & & Max & \\
\hline & & LPP & $\mathrm{P}_{\mathrm{det}}$ & PVR & LPP & $\mathrm{P}_{\mathrm{det}}$ & PVR \\
\hline 45 & MS & 65 & 100 & 105 & 20 & 36 & 30 \\
\hline $62^{a}$ & $\mathrm{C} 7 \mathrm{C}$ & 72 & 100 & 150 & 12 & 48 & 37 \\
\hline 26 & C5 C & 46 & 50 & 500 & 25 & 56 & 250 \\
\hline $60^{\mathrm{a}}$ & T7 C & 60 & 80 & 300 & 40 & 50 & 300 \\
\hline 43 & T4 I & 52 & 65 & 150 & 26 & 36 & 195 \\
\hline 63 & MS & 100 & 100 & 50 & 72 & 96 & 500 \\
\hline 36 & C5 C & 80 & 100 & 100 & 40 & 65 & 0 \\
\hline 45 & C6 C & 91 & 100 & 250 & 42 & 74 & 100 \\
\hline 51 & $\mathrm{~T} 8 \mathrm{C}$ & 100 & 100 & 325 & 10 & 30 & 5 \\
\hline 36 & C6 I & 47 & 50 & 200 & - & - & - \\
\hline
\end{tabular}

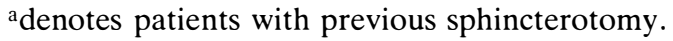
$\mathrm{C}=$ complete $\mathrm{I}=$ incomplete.

when it is deployed. The stent is preloaded in a special endoscopic introducer which allows the surgeon to visualize the prosthesis and urethra throughout the insertion process. When the Urolume stent is released from the introducer, it expands to its full 42 French diameter similar to a Chinese finger toy. If the length of the sphincter was greater than $25 \mathrm{~mm}$, two prostheses were placed with at least a $5 \mathrm{~mm}$ overlap between them to achieve adequate coverage of the urethral sphincter. When the repositioning or removal of the prosthesis was necessary, an alligator forceps was used endoscopically to grasp and pull it, resulting in lengthening and contraction of the prosthesis. The procedure was performed under spinal or local anesthesia.

All patients had postoperative pelvic films to confirm the location of the stent. The first few patients were observed for $48 \mathrm{~h}$ and the remainder for $24 \mathrm{~h}$ postoperatively before discharge. All patients were placed on a regimented bowel program and instructed by physical therapists to modify their transfer, in order to avoid increasing abdominal pressure and possibly dislodging the device. This regimen was followed for the first 3 months until the stent epithelialized. Urethral catheterization was also avoided postoperatively. All patients were followed with KUB, renal ultrasound, retrograde urethrogram, cystoscopy, and videouro- dynamics at 3, 6, and 12 months postoperatively. Videourodynamics were performed in a similar fashion pre- and postoperatively. Urine cultures via suprapubic aspirate and/or clean catch were obtained at 1,3 , 6 , and 12 months postoperatively.

\section{Results}

Ten patients 26-63 years old (mean 46 years) underwent placement of Urolume urethral wallstent for DSD. Serum creatinine, BUN and upper tracts were normal preoperatively in all patients. Urodynamic parameters are given in Table I. Four patients with previous sphincterotomy presented with autonomic dysreflexia, recurrent urinary tract infection (UTI) and the urodynamic values revealed high leak point pressure (LPP) as shown in Table I. Cystoscopy showed an open external urethral sphincter in these four patients; however, videourodynamic evaluation revealed persistent narrowing at the external urethral sphincter.

Initially of the 10 patients, eight required placement of one urethral stent and two required two overlapping stents to cover the sphincter. Two patients required suprapubic tube placements which were removed prior to discharge. Five patients had mild hematuria postoperatively which resolved sponta- 
neously within $24 \mathrm{~h}$. Postoperative urine collection was via condom catheter drainage.

On follow up all patients had normal upper tracts with no deterioration in renal function. Data of repeat videourodynamics at 12 months are given in Table I.

All patients had evidence of stent shortening and widening on pelvic films at 1 month as compared to immediate postoperative film. The length of the stent remained stable on further follow up. Stent migration in three patients resulted in partial uncoverage of the external sphincter with persistent high LPP. An additional stent was placed in each of these three patients that resulted in decrease in LPP and resolution of symptoms in one patient. Two patients continued to have autonomic dysreflexia despite placement of two overlapping urethral stents and adequate drainage of urine. One patient's stents were removed at his request. This patient failed to return for further follow up and is currently managed in another institution with a suprapubic catheter. The third patient had a low LPP on follow up urodynamic study, but continued to have autonomic dysreflexia. This patient underwent magnetic resonance imaging (MRI) of his abdomen and pelvis to further evaluate his symptoms. The MRI showed no abnormality except for gallstones. This patient has since been treated for his gallstones and his autonomic dysreflexia resolved.

One patient had continuing autonomic dysreflexia despite a wide open sphincter cystoscopically and a LPP of $37 \mathrm{~cm} \mathrm{H}_{2} \mathrm{O}$, and videourodynamic evaluation revealed smooth sphincter (bladder neck) dyssynergia. The patient was started on an alpha blocker that resulted in effective drainage and alleviation of dysreflexia and decline of his LPP to $25 \mathrm{~cm} \mathrm{H}_{2} \mathrm{O}$.

Follow up cystoscopy at 3 and 6 months showed that $80-100 \%$ of the stents were covered with urothelium respectively. There was no evidence of obstruction. No patient had symptomatic urinary tract infections although asymptomatic bacteriuria was documented in six. No patient reported change in frequency or rigidity of erection after stent placement.

\section{Discussion}

The use of external striated sphincterotomy to achieve improved bladder emptying in patients with DSD was first described by Emmett et al. ${ }^{7}$ Since then several approaches have been described, all having similar results and complication rates. The incision at the twelve o'clock position has become more popular secondary to its lower rate of erectile dysfunction. Sphincterotomy is associated with a high rate of complications including significant hemorrhage, urethral extravasation, infection, fistula formation, and erectile dysfunction. Lockhart et al reported a $25 \%$ rate of complication in a group of 60 patients who had sphincterotomy. ${ }^{2}$ Other alternative treatment options include chronic indwelling catheterization, pharmacologic therapy and urinary diversion. ${ }^{8-10}$ Pharmacologic treatment with muscle relaxing agents has not been proven to be effective. ${ }^{9}$ Use of botulinum A toxin is cumbersome and requires repeated treatments. To avoid some of these complications recent investigators have introduced the use of urethral stents in treatment of DSD.

Expandable stainless steel mesh stents were developed for use in the vascular system. Similar stents have been used in treatment of $\mathrm{BPH}$, urethral stricture and recently DSD with relatively long term success. We evaluated the use of the Urolume urethral stent in treatment of DSD. The Urolume stent is made of a superalloy mesh that expands and shortens similar to a Chinese finger toy. This characteristic of the prosthesis facilitates its insertion and removal under direct vision. When the prosthesis is deployed it expands and shortens, creating a 42 French diameter lumen. The elastic property and the radial force of the stent allow it to remain in place and overcome obstruction by the external sphincter. The large lumen created by the stent allows easy catheterization and cystoscopy after the stent is covered by the urothelium.

Our initial results in 10 patients with at least 12 months follow up have been encouraging. In addition to symptomatic relief, our goal was to achieve bladder emptying at a 
low pressure with LPP less than $40 \mathrm{~cm} \mathrm{H}_{2} \mathrm{O}$. In patients with myelodysplasia and neuropathic voiding dysfunction, the risk of upper tract deterioration is significantly increased when LPP is greater than $40 \mathrm{~cm} \mathrm{H}_{2} \mathrm{O} .{ }^{11} \mathrm{No}$ one has developed symptomatic urinary tract infections. Autonomic dysreflexia has resolved in six of eight patients who had it preoperatively. The follow up urodynamics shows persistent DSD, detrusor instability and urge incontinence; however, eight patients have a lower LPP, $\max \mathrm{P}_{\text {det }}$ and post void residual (PVR). When the LPP remained high, an identifiable anatomic or functional obstruction was found. The stent lowered the LPP in eight patients. All patients have normal upper urinary tracts and no deterioration in renal function in short term follow up was noted. The stents shortened during the first month post insertion as compared to immediately postoperatively, then stabilized in length as observed on the follow up pelvic films. The stent exerts radial forces that overcome the forces generated by the external sphincter. This leads to dilation and relaxation of the external sphincter. As the external sphincter relaxes, it allows the stent to further expand and shorten. This characteristic of the stent allows it to embed into the tissue, thereby stabilizing it and preventing further shortening of the stent.

The stent shortened in all patients except in one in whom it migrated. Since the stent shortens during the first month post insertion, it is necessary to ensure that it covers at least $5 \mathrm{~mm}$ distal and proximal to the sphincter. Stent shortening may result in partial uncoverage of the sphincter simulating migration. The same factors that influence the shortening of the stent may also effect its migration. If the external sphincter is very spastic and has a very high tone, it can potentially overcome the radial forces exerted by the stent, leading to stent migration. Initially, in patients who only had one stent placed, two had stent migration. They both had difficulty with drainage, and urodynamic evaluation revealed high LPP with obstruction at the external urethral sphincter. The LPP was lower than preoperatively but higher than in the immediate postoperative period. Cystoscopy revealed that a small ring of the external sphincter was not covered by the stent. After a second stent was placed, both patients had improvement in bladder drainage at a lower detrusor pressure. This observation illustrates several interesting points. The sphincter muscle units appear to act in a circular rather than longitudinal fashion. The periurethral component of the intrinsic sphincter is longitudinal; however, the extrinsic component is circular. The stent keeps the longitudinal component open; however, for the stent to properly keep the circular component open, it needs to cover the entire length of the sphincter. The other possible conclusion is that the sphincter muscle is composed of multiple components that act individually. When one or more components are not covered by the stent, that component can act independently and lead to persistent obstruction.

Anatomically there are three potential locations in the urethra that can lead to obstruction: the bladder neck, prostatic urethra, and the membranous urethra, and obstruction may occur at one or more sites. This was seen in one patient who continued to have autonomic dysreflexia and difficulty with bladder drainage even though the sphincter area was wide open. Videourodynamic evaluation revealed bladder neck dyssynergia and cystoscopy showed an open external urethral sphincter and small prostate. He was treated with an alpha blocker, and his symptoms improved. The retrospective review of his videourodynamic study demonstrated a persistently closed bladder neck and DSD. Previous reports have suggested that bladder neck hypertrophy may evolve after stent placement that may lead to obstruction and elevated LPP. There was no evidence of bladder neck hypertrophy, in our patient; rather, a combination of bladder neck dyssynergia and DSD that was undiagnosed preoperatively as the cause of the patient's symptoms. We were able only to reach this conclusion after retrospective analysis of the videourodynamic that underscores the importance of using imaging studies in addition to urodynamic studies in the evaluation of these patients.

Follow up cystoscopies revealed that the 
stents were epithelialized within 3-6 months post insertion. Milroy also reported similar results when he used the stents to treat urethral strictures. ${ }^{8}$ As the stent becomes embedded in the tissue, urothelium grows over its entire surface.

All patients wear a condom catheter postoperatively for urine collection. The main concern with the long term use of a condom catheter is that it may lead to persistent bacteriuria and result in progressive destruction of the upper urinary tract. Sotolongo et al followed 56 SCI patients on long term condom catheter drainage over a period of 5 years. ${ }^{12}$ No patient had evidence of upper tract deterioration when the serum creatinine and upper tract studies were followed. Six of our patients had asymptomatic bacteriuria postoperatively, documented by urine cultures obtained via suprapubic aspiration or clean catch specimen; however, none have developed symptomatic urinary tract infection. Our results compare favorably with those recently reported by McInerney, ${ }^{13}$ Shaw, ${ }^{14}$ and Chancellor $^{15}$ in the use of the urethral stents in the treatment of DSD. Their experience with this device extends up to 3 years and no long term adverse reactions have been observed. They reported a low complication rate and high success in treatment of DSD. ${ }^{13-15}$

When a foreign object is placed inside the body, there is always concern regarding its long term safety and its potential for carcinogenicity. The European experience with the stent extends up to 3 years and there have been no reported complications. ${ }^{13,14}$ We have not observed any adverse effects during our short term follow up; however, long term observation is necessary to establish its safety.

In conclusion, we believe that the Urolume urethral stent offers an acceptable option for the treatment of DSD. It can be used in place of sphincterotomy or in patients who have had poor outcome after sphincterotomy. The prosthesis is easy to insert and remove when necessary, with minimal morbidity. Bladder drainage is accomplished easily, and there is no adverse effect on erectile function. Even though, as part of this protocol, we observed our patients overnight, the procedure can be performed on an outpatient basis. Our short term results are encouraging; however, long term follow up is necessary to establish its safety and efficacy.

\section{References}

1 Hackler RH (1977) A 25 year prospective mortality study in spinal cord injured patient: Comparison with long-term living paraplegic. J Urol 117: 486-488.

2 Lockhart JL, Pow-Sang JM (1989) Indications and problems with external urethral sphincterotomy. Probl Urol 3: 44-50.

3 Sigwart U, Puel J, Mirkovitch V et al (1987) Intravascular stents to prevent occlusion and restenosis after transluminal angioplasty. $N$ Engl J Med 316: 701-706.

4 Rousseau H, Puel J, Joffre F et al (1987) Self expanding endovascular prosthesis: An experimental study. Radiology 164: 709-714.

5 Oesterling JE (1993) Urologic applications of a permanent epithelializing urethral endoprosthesis. Urology (Suppl) 41: 10-18.

6 Oesterling JE (1991) A permanent, epithelializing stent for the treatment of benign prostatic hyperplasia: Preliminary results. J Androl 12: 423-428.

7 Emmett JL, Daut RV, Dunn JH (1948) Role of the external urethral sphincter in the normal bladder and cord bladder. J Urol 59: 439-454.

8 DeVivo MJ, Rutt RD, Black KJ et al (1992) Trends in spinal cord injury demographics and treatment outcome between 1973 and 1986. Arch Phys Med Rehabil 73: 424-430.

9 Hacken HJ, Krucker V (1977) Clinical and laboratory assessment of the efficacy of baclofen (Lioresil) on urethral sphincter spasticity in patients with traumatic paraplegia. Eur Urol 3: 237-240.

10 Dykstra DD, Sidi AA (1990) Treatment of detrusor sphincter dyssynergia with botulinum A Toxin: A double-blind study. Arch Phys Med Rehabil 71: 24-26.

11 McGuire EJ, Woodside JR, Borden TA, Weiss RB (1981) Prognostic value of urodynamic testing in myelodysplastic patients. J Urol 126: 205-209.

12 Sotolongo JR, Koleilat N (1990) Significance of asymptomatic bacteriuria in spinal cord injury patients on condom catheter. J Urol 143: 979-980. 
13 McInerney PD, Vanner TF, Harris SA, Stephenson TP (1991) Permanent urethral stents for detrusor sphincter dyssynergia. Br J Urol 67: 291-294.

14 Shaw PJ, Milroy EJ, Timoney AG et al (1990) Permanent external striated sphincter stents in patients with spinal injuries. Br J Urol 66: 297-302.

15 Chancellor MB (1991) Urinary sphincter prosthesis. Curr Surg Tech Urol 4: 1-6. 